\title{
Optical and Electrical Noise Characteristics of Side Emitting LEDs
}

\author{
B. ŠAulys ${ }^{a, *}$, V. KorniJČUK ${ }^{a}$, J. MAtukas $^{a}$, V. PAlenskis $^{a}$, \\ S. Pralgauskaité $\dot{1}^{a}$ And K. GlemŽA ${ }^{b}$ \\ ${ }^{a}$ Radiophysics Dep., Vilnius University, Saulètekio 9 (III), 10222 Vilnius, Lithuania \\ ${ }^{b}$ Theoretical Physics Dep., Vilnius University, Saulètekio 9 (III), 10222 Vilnius, Lithuania
}

\begin{abstract}
Low frequency noise characteristics of nitride based blue side emitting diodes have been investigated. It is shown that investigated devices distinguish by $1 / f^{\alpha}$-type optical and electrical fluctuations caused by various generation-recombination processes through defects formed generation-recombination centers. At higher frequencies optical shot noise due to random photon emission prevails $1 / f^{\alpha}$-type spectrum. The results have shown that low frequency optical and electrical noises are strongly correlated at small current region, but at higher forward current not correlated noise components dominate. Lenses and secondary optics of the investigated devices do not influence output light.
\end{abstract}

PACS: $72.70 .+\mathrm{m}, 74.40 .-\mathrm{n}, 85.60 . \mathrm{Jb}$

\section{Introduction}

Nowadays light emitting diodes (LEDs) are common in a variety of electronic equipment. Huge attention is given to the LED characteristics improvement $[1,2]$.

Semiconductor light emitting device radiation spectrum has moved to the shorter wavelengths, when nitride-based structures were started using for the optoelectronic devices. However, nitride layers introduce additional defects in the multilayer structure due to large number of threading dislocations [3]. Also phosphor layer used in the white LEDs can be additional source of device degradation. Understanding of physical processes that take place in different parts of the device is necessary for the LED quality and reliability improvement. It is well known that noise investigation gives valuable information on physical processes that take place in semiconductor materials and structures [4] and is useful for the evaluating quality of semiconductor devices and predicting their lifetime $[4,5]$.

In the side emitting diode the light goes upwards and is collimated and redirected $90^{\circ}$ by a conical reflector to exit in the horizontal plane. Special secondary optics should be used that fits over a Batwing- or Lambertian-type device. Investigation of the side emitting diodes enables us to separate physical processes that take part in different parts of the device and gives better understanding, which part of the device influences its degradation foremost.

The aim of the work was to investigate the side emitting diode low frequency noise characteristics, to find

* corresponding author; e-mail: bronius.s@lokmis. It out noise features that indicate LED degradation, and to clear up physical processes that take part in different parts of the LED and influence their lifetime.

\section{Investigated LEDs and measurement technique}

Investigated devices were nitride-based side emitting diodes radiating blue (470 $\mathrm{nm}$ ) light. Radiation pattern of the diode has toroid form and intensity of the light has a peak at 75-85 degrees off the optical axis.

Optical and electrical noise characteristics (electrical noise (terminal voltage fluctuation of the light emitting diode) spectral density, optical noise (LED light output power fluctuation) spectral density, mutual correlation factor between optical and electrical fluctuations and mutual correlation factor between two optical signals detected at the opposite directions of the LED radiation pattern) of the investigated LEDs have been measured. Therefore, for the side emitting diode optical noise investigation two photodiodes have been aimed precisely at the points of the peak intensity of the diode (Fig. 1).

Noise power spectral densities of electrical and optical fluctuations can be decomposed to the statistically independent components of $1 / f, 1 / f^{\alpha}$, Lorentzian type, and shot noise spectra [6]:

$$
S(f)=\frac{A_{1 / f}}{f}+\frac{A_{1 / f^{\alpha}}}{f^{\alpha}}+\frac{A_{\mathrm{gr}} \tau}{1+(2 \pi f \tau)^{2}}+S_{\text {shot }},
$$

where quantities $A_{j}$ define the intensities of noise components. At the first sight fluctuation of LED output power should repeat terminal voltage fluctuation. But not all 


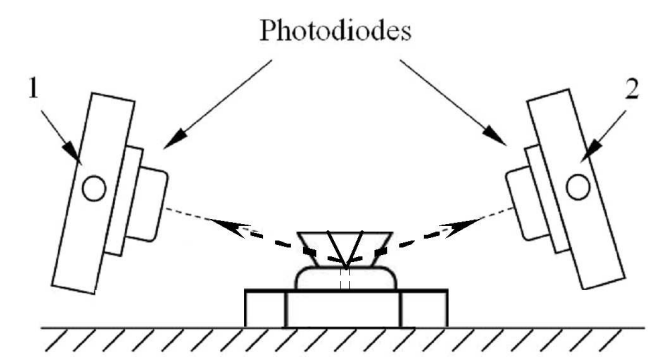

Fig. 1. Side emitting diode investigation scheme ( 1 and 2 are photodiodes rotation axis).

low-frequency electrical fluctuations are completely correlated with optical fluctuations: contact or electrical noises in passive layers of LED do not cause the emitted light intensity fluctuations, i.e., every spectral component of low-frequency electrical noise can be presented as a sum of correlated and uncorrelated parts

$$
\begin{aligned}
& S_{j \Sigma}(f)=S_{j k=100 \%}(f)+S_{j k=0 \%}(f) \\
& \quad=d_{j} S_{j}(f)+\left(1-d_{j}\right) S_{j}(f) ;
\end{aligned}
$$

here quantity $d_{j}$ shows what part of spectral component $S_{j}(f)$ of electrical noise is related with emitted light intensity fluctuations, index $j$ describes different noise components.

\section{Results and discussion}

Typical electrical and optical noise characteristics for the investigated LEDs are presented in Figs. 2 and 3. Electrical noise of the investigated LEDs is mainly characterized by $1 / f^{\alpha}$-type fluctuations (part (a) in Fig. 2). The source of $1 / f^{\alpha}$-type noise in semiconductor devices generally is caused by the superposition of charge carrier generation-recombination (GR) and capture processes through GR and capture centers with widely distributed relaxation times [7]. These centers are formed by different defects, dislocations and imperfections in the device structure, and can be located in the active area or other layers and interfaces of the LEDs. More intensive electrical noise at small current and its decrease with forward current increasing (part (a) in Fig. 3) can be explained by defects in LED structure: at small current conditions current flows through the narrow channels formed by the defects [7], and the electron capture or release events in these defects lead to the large random modulation of flowing current. As forward current density increases through the other part of the device cross-section, the influence of the defect decreases due to shunting effect.

Fluctuations of output light power for investigated device show $1 / f^{\alpha}$-type spectrum at low frequencies and "white" noise at frequencies above $1 \mathrm{kHz}$ (part (b) in Fig. 2). 1/ $f^{\alpha}$-type optical fluctuations are caused by the modulation of charge carrier number in the active region due to flowing current fluctuations. Optical fluctuations with "white" spectrum are attributable to the

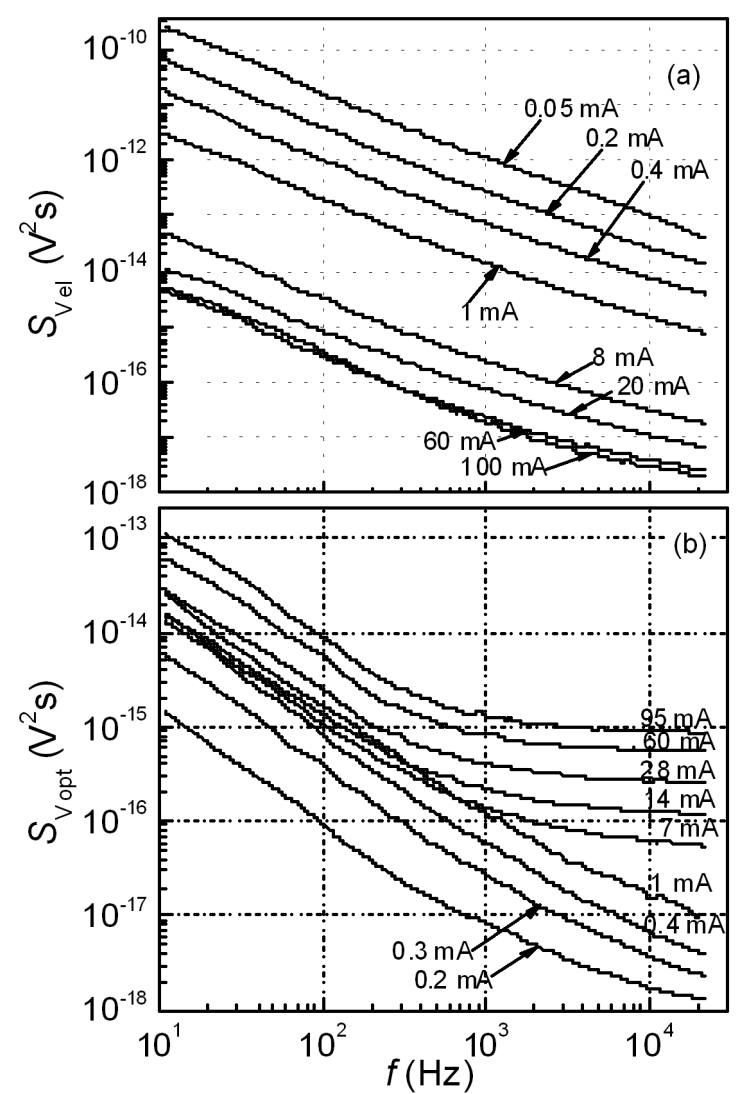

Fig. 2. Electrical (a) and optical (b) noise spectra of side emitting diode at different forward currents.

shot noise due to emitted photons. Optical noise spectral density increases with forward current increase (part (b) in Fig. 3) as light output power increases.

Fluctuation of LED output power is partly correlated with electrical noise as presented in Figs. 3 (part (c)) and 4 . At low frequencies $(<100 \mathrm{~Hz})$, where $1 / f^{\alpha}$-type fluctuations dominate both in optical and electrical noise, at small current region $(<10 \mathrm{~mA})$ optical fluctuation repeats flowing current fluctuation, i.e. optical and electrical $1 / f^{\alpha}$-type noises are strongly correlated (Fig. 4). There is some defective regions in the investigated device active layer that modulate flowing current herewith modulate injected charge carriers number and emitted photon number. As forward current increases current flow redistributes and not correlated optical and electrical noise components start dominating (Fig. 4). At higher current region significant part of the injected current flows through the peripheral areas of the LED and does not participate in the photons emission process.

Correlation factor between two optical signals detected at opposite sides of the side emitting diode radiation pattern have been measured in every one-octave frequency band (Fig. 5). Close to $100 \%$ correlation was observed and it is true to say that light emission from the active region goes from one point and lenses and secondary optics do not influence the pervasive light. 


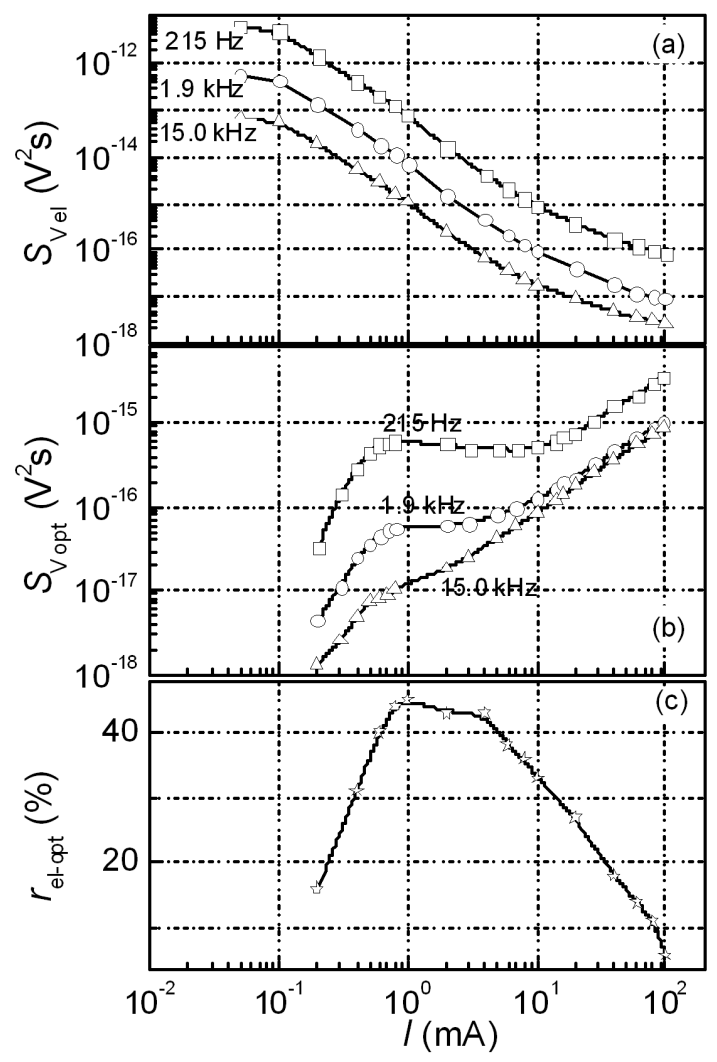

Fig. 3. Electrical (a) and optical (b) spectral density at three different frequencies and mutual correlation factor between optical and electrical fluctuations ((c) in the frequency range from $20 \mathrm{~Hz}$ to $20 \mathrm{kHz}$ ) dependences on forward current.

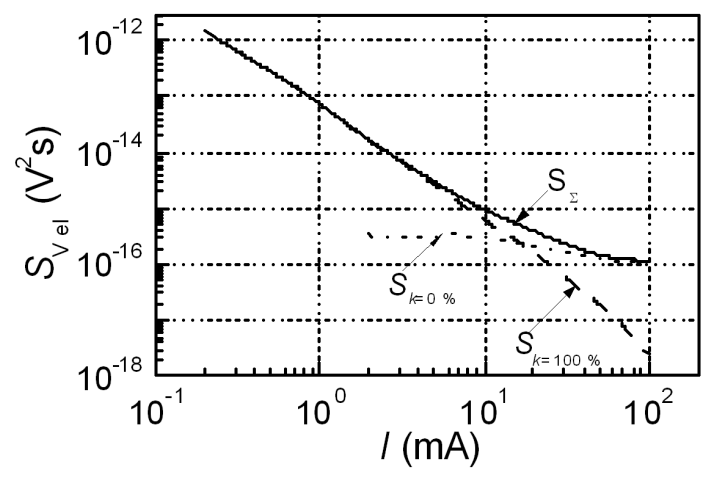

Fig. 4. Electrical noise spectral density $\left(S_{\Sigma}\right)$ decomposition (according to Eq. (2)) into fully correlated with optical noise $\left(S_{k=100 \%}\right)$ and uncorrelated $\left(S_{k=0 \%}\right)$ components $(f=215 \mathrm{~Hz})$.

\section{Conclusions}

Optical and electrical $1 / f^{\alpha}$-type fluctuations of the investigated light emitting diodes (LEDs) at low frequen-

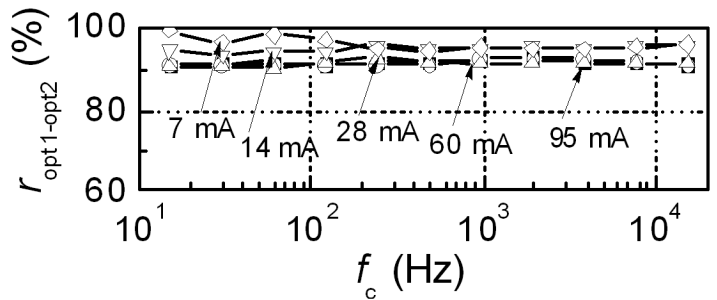

Fig. 5. Mutual correlation factor between two optical signals detected at the opposite points of the side emitting diode radiation pattern dependence on octave central frequency at different forward currents.

cies are caused by the superposition of GR processes through defects created charge carrier GR and capture centers. Optical noise with "white" spectrum at higher frequencies is related to the shot noise due to random photon emission.

At small current region $1 / f^{\alpha}$-type optical and electrical noise components are strongly correlated: current flowing through the active area unambiguously modulates emitted photon number. At higher forward current uncorrelated optical and electrical noise components prevail: injected current flow through the peripheral device regions has significant influence on the electrical noise signal but does not influence output light power.

As has shown close to $100 \%$ correlation factor between optical signals detected at opposite points of the radiation pattern lenses and secondary optics do not influence the output light.

\section{References}

[1] H.Y. Ryu, T. Sakong, J.K. Son, Electron. Lett. 45, 1318 (2009).

[2] H.J. Chung, R.J. Choi, M.H. Kim, J.W. Han, Y.M. Park, Y.S. Kim, H.S. Paek, C.S. Sone, Y.J. Park, J.K. Kim, E.F. Schubert, Appl. Phys. Lett. 95, 241109 (2009).

[3] Y.-J. Lee, H.-C. Kuo, T.-C. Lu, S.-C. Wang, K.W. Ng, K.M. Lau, Z.-P. Yang, A.S.-P. Chang, S.-Y. Lin, J. Light. Technol. 26, 1455 (2008).

[4] D. Ursutiu, B.K. Jones, Semicond. Sci. Technol. 11, 1133 (1996).

[5] S. Pralgauskaite, V. Palenskis, J. Matukas, J. Petrulis, G. Kurilcik, Fluct. Noise Lett. 7, L367 (2007).

[6] V. Palenskis, J. Matukas, S. Pralgauskaitè, B. Šaulys, Fluct. Noise Lett. 9, 179 (2010).

[7] B.K. Jones, Electron. Electron Phys. 87, 201 (1994). 